\title{
Evaluation of Chromium Speciation and Transport Characteristics in the Hanford Site 100D and $100 \mathrm{H}$ Areas
}

Prepared for the U.S. Department of Energy Office of Environmental Restoration and Waste Management

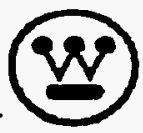
Westinghouse Hanford Company Richland, Washington

Management and Operations Contractor for the

U.S. Department of Energy under Contract DE-ACO6-87RL10930

Copyright Liconse By acceptence of this article, the publisher and/or rocipient acknowledges the

U.S. Government's right to retain a nonexdusive, royalty-free license in end to any copyright covering this peper. 


\title{
Evaluation of Chromium Speciation and Transport Characteristics in the Hanford Site $100 \mathrm{D}$ and $100 \mathrm{H}$ Areas
}

\author{
E. C. Thornton
}

Westinghouse Hanford Company

J. E. Amonette

J. Olivier

D. L. Huang

Pacific Northwest Laboratory

Date Published

August 1995

To Be Presented at

1st Symposium

Hydrogeology of Washington State

Olympia, Washington

August 28-30, 1995

Prepared for the U.S. Department of Energy

Office of Environmental Restoration and

Waste Management

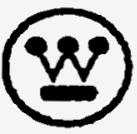

Westinghouse

P.O Box 1970

Hanford Company Richland, Washington

Management and Operations Contractor for the

U.S. Department of Energy under Contract DE-AC06-87RL10930

Copyright License By acceptance of this artide, the publisher and/or recipient acknowlodges the

U.S. Government's right to retain a nonexdusive, royalty-free license in and to any copyright covering this peper. 


\section{DISCLAIMER}

Portions of this document may be illegible in electronic image products. Images are produced from the best available original document. 


\title{
EVALUATION OF CHROMIUM SPECIATION AND TRANSPORT CHARACTERISTICS
} IN THE HANFORD SITE 1000 AND 10OH AREAS

\author{
E.C. Thornton, Westinghouse Hanford Company \\ J.E. Amonette, J.A. 0livier, and D.L. Huang, Pacific Northwest Laboratory
}

Fjeld and laboratory investigations have been conducted to define the fate and transport characteristics of chromium contamination present in the $100 \mathrm{D} / \mathrm{H}$ Areas of the Hanford Site. This information is relevant to assessing the impact of the release of hexavalent chromium to the Columbia River associated with the discharge of groundwater from the Hanford unconfined aquifer. Included in this study was the determination of the concentration and aqueous speciation of chromium in the unconfined aquifer, and an assessment of potential changes in speciation as groundwater passes through the river/aquifer transition zone and mixes with the Columbia River.

The fate of chromium was évaluated through the determination of chromium speciation of water samples collected from the Hanford unconfined aquifer, from seeps along the river, and from the river itself. The potential for uptake of chromium in the riverbed and riverbanks resulting from interaction with organic matter and oxide components associated with sediments was assessed by characterization of riverbank sediment samples. Mixing tests involving chromium-contaminated groundwater and river water were also conducted to determine if alteration of chromium speciation could occur subsequent to entering the Columbia River. In addition, groundwater plume maps were prepared from existing monitoring data of the 100-D and 100-H Areas to provide information regarding groundwater flow paths and potential chromium sources.

The results of this study indicate that chromium present within the Hanford unconfined aquifer of the 100-D and 100-H Areas is predominantly in the hexavalent oxidation state. Chromium is apparently relatively stable in the oxidized form owing to the lack of organic matter within the aquifer. A portion of the chromium is removed as groundwater passes through the river/aquifer transition zone due to reduction and precipitation associated with sediment/water interaction processes. Chemical data collected from seep water samples, however, suggests that most of the hexavalent chromium ultimately discharges into the Columbia River. Dilution of hexavalent chromium subsequently occurs during the mixing of groundwater and river water, with relatively little change taking place in speciation. 


\section{OVERVIEW}

- Objectives of Study

- Define chromium speciation of $100 \mathrm{D} / \mathrm{H}$ groundwater

- Assess potential changes in chromium concentration and speciation as groundwater flows through aquifer/ river interface and mixes with Columbia River

- Approach

- Define current chromium distribution patterns

- Determine chromium speciation of water samples

- Characterize riverbank sediments

'_- Perform groundwater/river laboratory mixing tests 


\section{ENVIRONMENTAL CHEIMISTRY OF CHROMIUM}

- Chromate or Hexavalent Chromium

- $\mathrm{CrO}_{4}{ }^{2-}$ is predominant hexavalent chromium species

- Toxic and mobile

- Utilized as corrosion inhibitor and decontaminant

- Reduction from Hexavalent to Trivalent Oxidation State

$$
\mathrm{CrO}_{4}{ }^{2-}+8 \mathrm{H}^{+}+3 \mathrm{e}^{-} \rightarrow \mathrm{Cr}^{3+}+4 \mathrm{H}_{2} \mathrm{O}
$$

- Important Reductants in Environment

- Organic matter

- Ferrous iron

- Trivalent Chromium

- Nontoxic, natural form of chromium

$1,-\mathrm{Cr}^{3+}$ immobilized by precipitation and adsorption

$$
\mathrm{Cr}^{3+}+3 \mathrm{OH}^{-} \rightarrow \mathrm{Cr}(\mathrm{OH})_{3}
$$




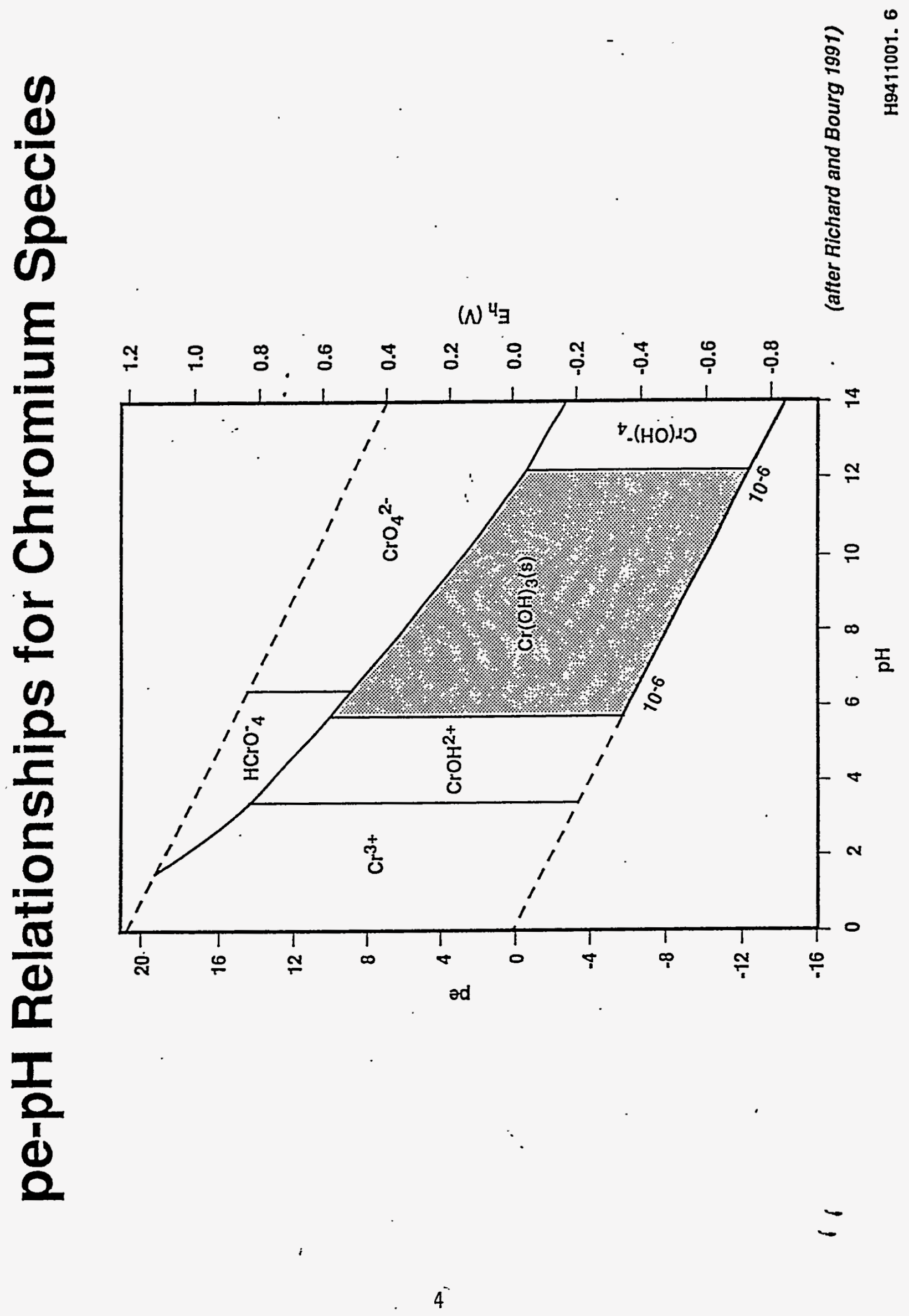




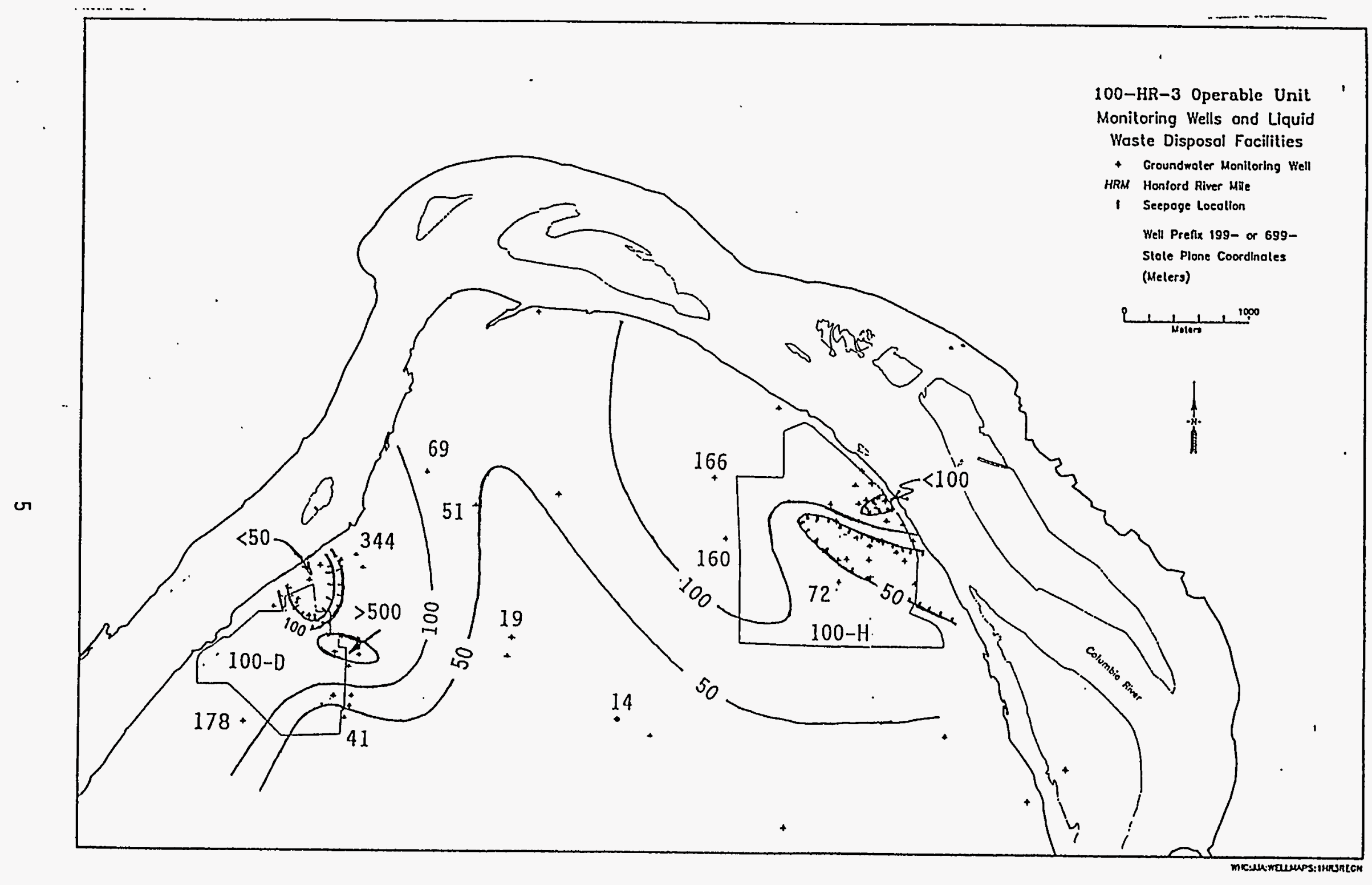

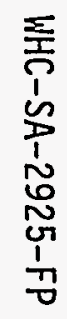

Chromium Distribution in the Hanford Site Unconfined Aquifer of the 100-D and 100-H Areas. Data shown is total chromium concentration values in $\mathrm{ppb}$ for filtered groundwater samples collected in October of 1992. 


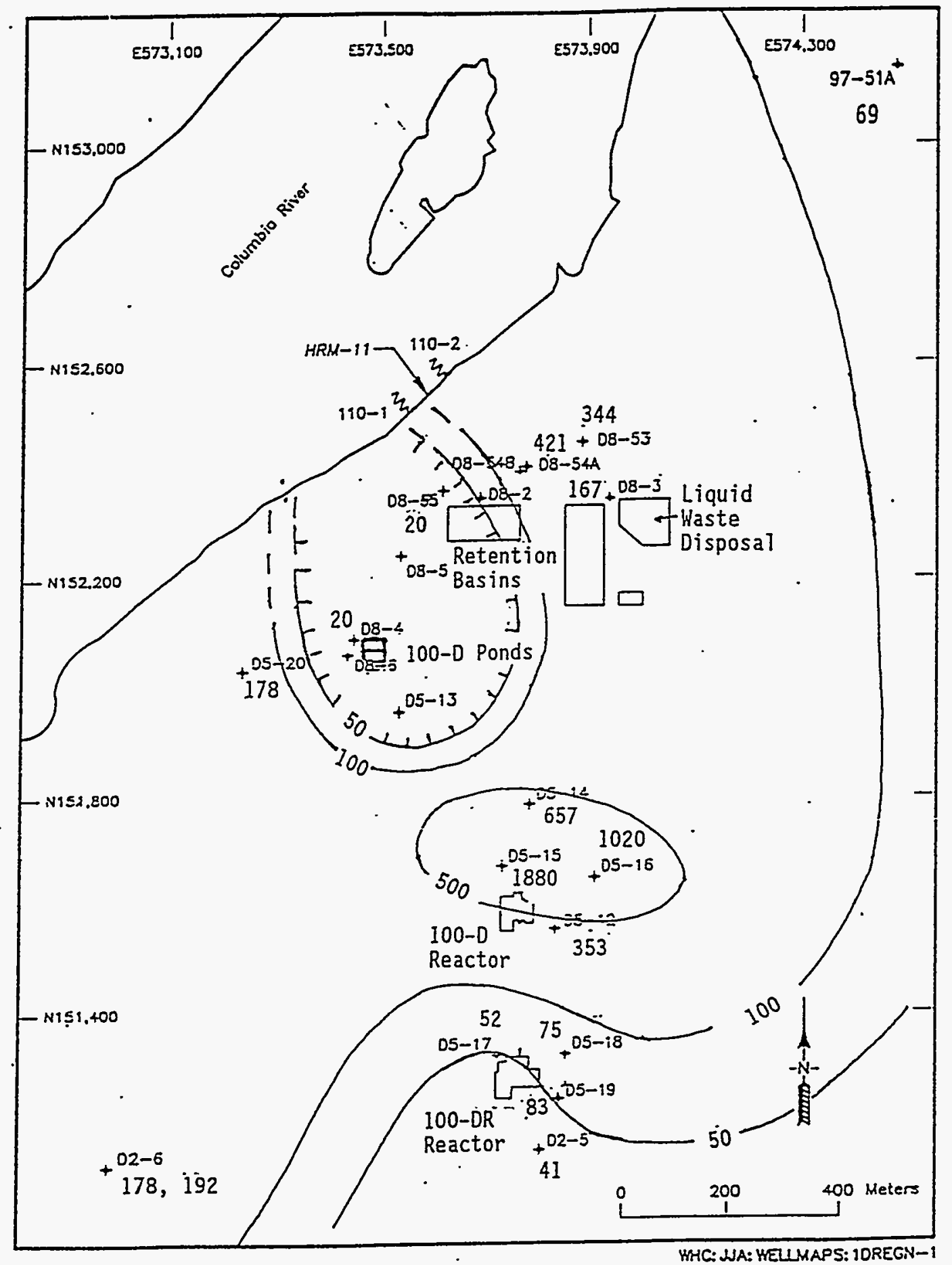

Chromium Distribution in the 100-D Area. Data shown is total chromium concentration values in $\mathrm{ppb}$ for filtered groundwater samples collected in October of 1992. 


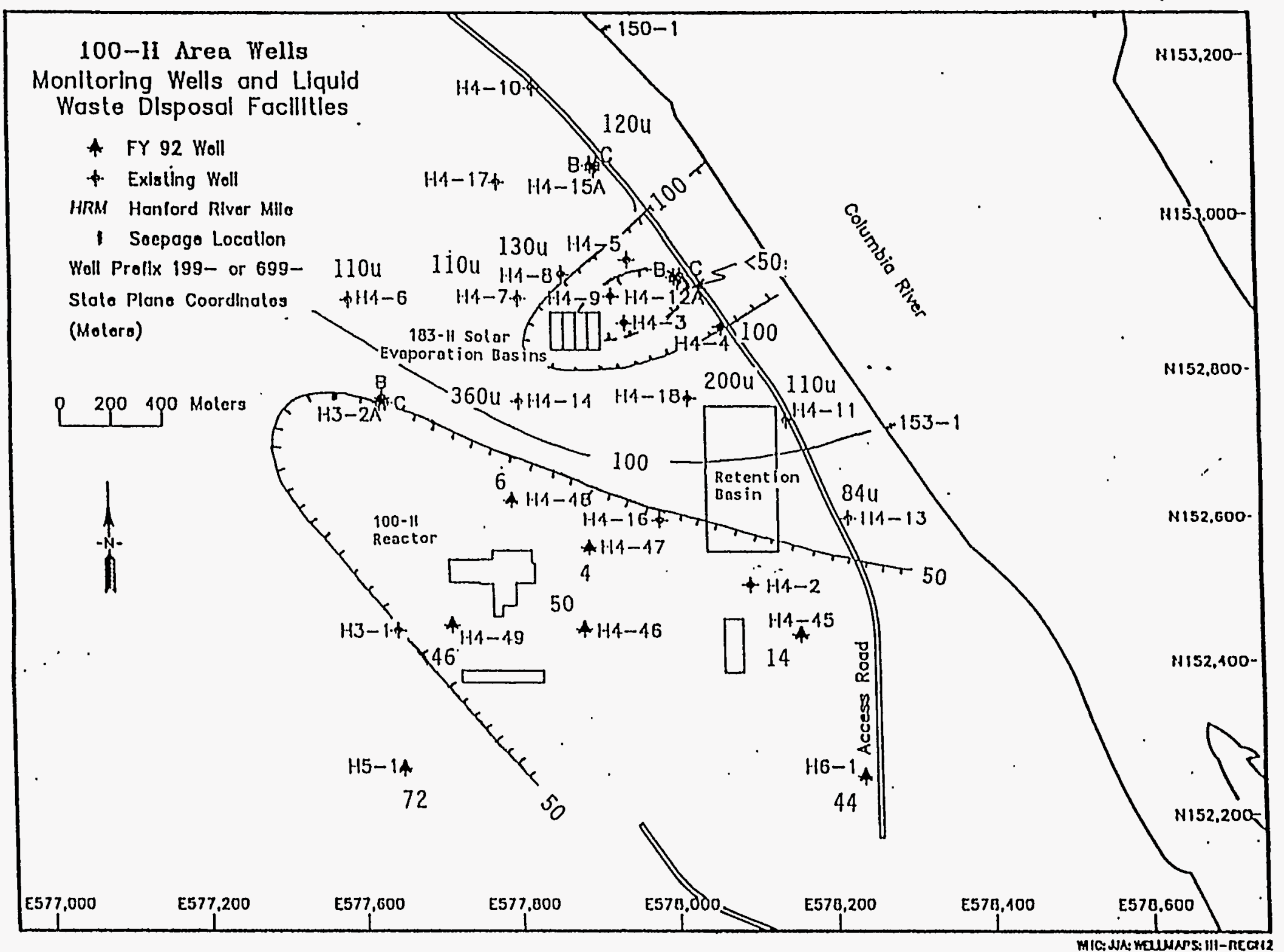

Chromium Distribution in the 100-H Area. Data shown is total chromium concentration values in $\mathrm{ppb}$ for filtered groundwater samples collected in September through November of 1992, and unfiltered samples (indicated by u suffix) collected in October of 1992. 
WHC-SA-2925-FP

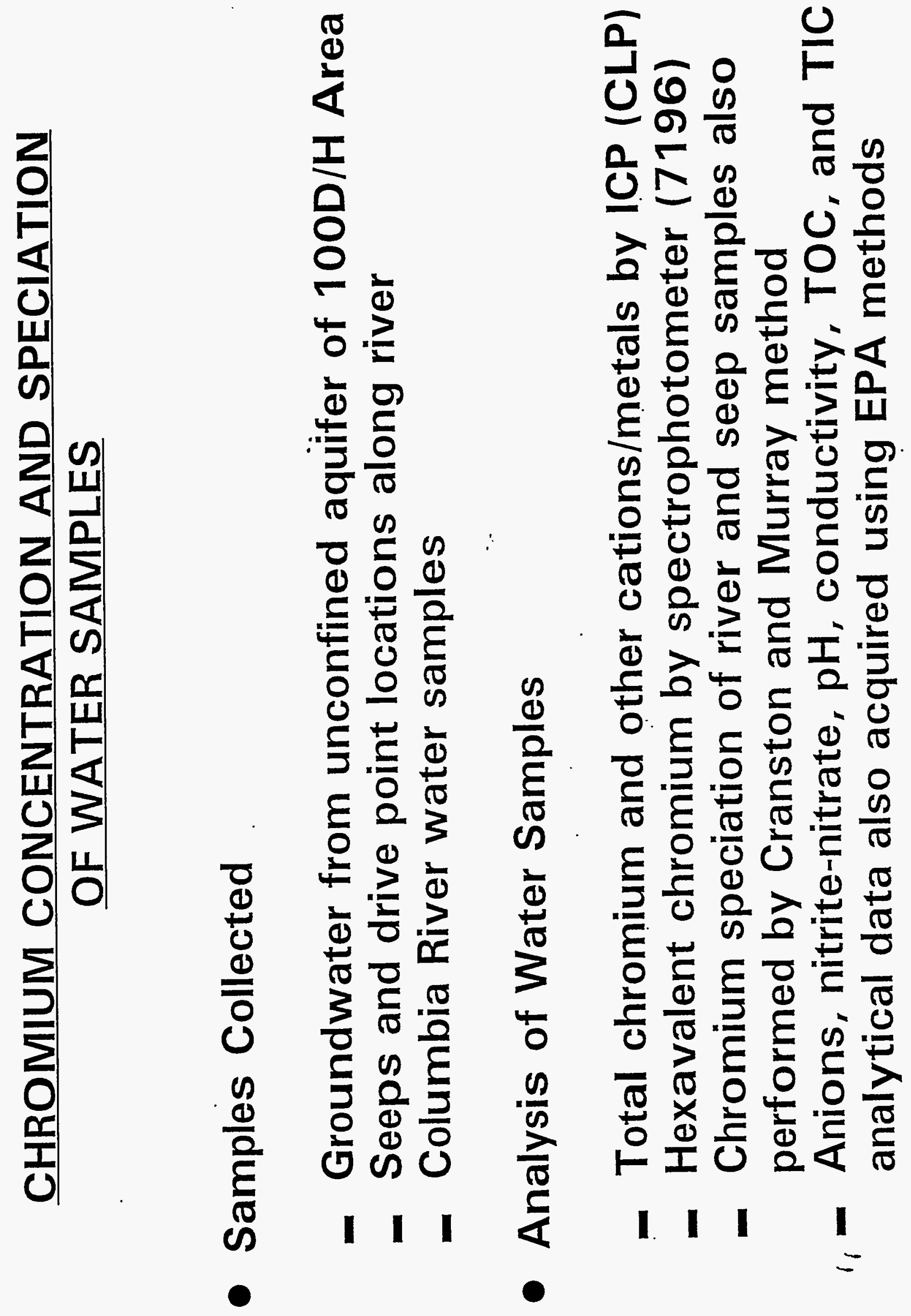


Hexavalent and Total Chromium Analytical Data (mg/L) for Groundwater Samples Collected in the 100-D and 100-H Areas.

\begin{tabular}{|c|c|c|c|c|c|}
\hline We11 \# & $\begin{array}{l}\text { Date } \\
\text { Time } \\
\end{array}$ & $\begin{array}{l}\operatorname{Cr}(V I), 7196 \\
\text { unfiltered }\end{array}$ & $\begin{array}{l}\mathrm{Cr}(\mathrm{VI}) \\
\mathrm{Hach}\end{array}$ & $C r_{T}, I C P$ & $\begin{array}{l}\mathrm{Cr}_{\mathrm{T}} \\
\mathrm{Hach}\end{array}$ \\
\hline D5-15 & $\begin{array}{l}7 / 13 / 93 \\
08: 47\end{array}$ & -- & $1.26 f$ & -- & $\begin{array}{l}1.85 \mathrm{uf} \\
1.80 \mathrm{f}\end{array}$ \\
\hline $\mathrm{H} 4-4$ & $\begin{array}{l}7 / 13 / 93 \\
09: 40 \\
\end{array}$ & -- & $0.05 f$ & 0.075 uf B08QZO & $\begin{array}{l}0.05 u f \\
0.05 f \\
\end{array}$ \\
\hline $\mathrm{H} 4-3$ & $\begin{array}{l}7 / 13 / 93 \\
10: 15 \\
\end{array}$ & :- & $0.13 f$ & 0.180 uf B08QYg & $\begin{array}{l}0.18 u f \\
0.16 f \\
\end{array}$ \\
\hline$D 5-15$ & $\begin{array}{l}5 / 13 / 94 \\
09: 20\end{array}$ & 1.65 BOBML3 & $\begin{array}{l}1.1 \mathrm{f} \\
1: 2 \mathrm{f} \\
1: 45 \mathrm{f}\end{array}$ & $\begin{array}{l}1.59 u f \text { BOBMLI } \\
1.40 f \text { BOBML2 }\end{array}$ & $1.45 \mathrm{f}$ \\
\hline $\begin{array}{l}\text { D5-15 } \\
\text { (dup) }\end{array}$ & $\begin{array}{l}5 / 13 / 94 \\
09: 25 \\
\end{array}$ & 1.66 BOBML6 & $\begin{array}{l}1.6 f \\
1.3 f\end{array}$ & $\begin{array}{l}1.65 \text { uf BOBML4 } \\
1.50 f \quad \text { BOBML5 } \\
\end{array}$ & $1.3 \mathrm{f}$ \\
\hline D5-16 & $\begin{array}{l}5 / 13 / 94 \\
11: 15 \\
\end{array}$ & -- & $\begin{array}{l}0.78 \mathrm{f} \\
0.81 \mathrm{f} \\
\end{array}$ & -- & $0.80 \mathrm{f}$ \\
\hline D8-53 & $\begin{array}{l}5 / 13 / 94 \\
12: 00\end{array}$ & 0.41 BOBML9 & $\begin{array}{l}0.32 f \\
0.35 f \\
0.34 u f \\
0.38 u f \\
\end{array}$ & $\begin{array}{l}0.329 \text { uf } \\
0.368 \mathrm{~B} M L 7 \\
0.30 \mathrm{f} \text { BML8 }\end{array}$ & $\begin{array}{l}0.36 f \\
0.35 u f\end{array}$ \\
\hline $\mathrm{H} 4-14$ & $\begin{array}{l}5 / 13 / 94 \\
13: 30 \\
\end{array}$ & $\angle 0.05$ ВОВМM2 & $\begin{array}{l}0.04 \mathrm{f} \\
0.04 \mathrm{f} \\
\end{array}$ & $\begin{array}{l}0.053 \text { uf BOBMMO } \\
0.051 \mathrm{f} \text { BOBMMI } \\
\end{array}$ & $0.03 f$ \\
\hline H4-1I & $\begin{array}{l}5 / 13 / 94 \\
14: 22 \\
\end{array}$ & $<0.05$ BOBMM5 & $\begin{array}{l}0.06 f \\
0.06 f\end{array}$ & $\begin{array}{l}0.064 \text { uf } \mathrm{BOBMM3} \\
0.066 \mathrm{f} \quad \mathrm{BOBMM4} \\
\end{array}$ & $0.05 f$ \\
\hline $\mathrm{H} 4-12 \mathrm{C}$ & $\begin{array}{l}5 / 13 / 94 \\
15: 00 \\
\end{array}$ & -- & $\begin{array}{l}0.26 f \\
0.27 f \\
\end{array}$ & -- & $0.24 f$ \\
\hline $\begin{array}{l}\text { Equipment } \\
\text { BTank }\end{array}$ & $\begin{array}{l}5 / 13 / 94 \\
16: 00 \\
\end{array}$ & $<0.05$ BOBMM 8 & $\begin{array}{l}0.00 f \\
0.00 f \\
\end{array}$ & $\begin{array}{l}\leq 0.004 \text { uf } \text { BOBMM6 } \\
\leq 0.004 \mathrm{f} \quad \text { BOBMM7 } \\
\end{array}$ & $0.00 f$ \\
\hline
\end{tabular}

WHC sample numbers are indicated by BO prefix.

$f=$ filtered sample

uf $=$ unfiltered sample

-- not analyzed

A11 samples analyzed by EPA SW-846 Method 7196 were unfiltered. 


\section{Hexavalent and Total Chromium Analytical Data (mg/L) for Seep Samples Collected in the 100-D and 100-H Areas.}

\begin{tabular}{|c|c|c|c|c|}
\hline Location & $\begin{array}{l}\text { Date } \\
\text { Time }\end{array}$ & $\begin{array}{l}\operatorname{Cr}(V I), 7196 \\
\text { unfiltered }\end{array}$ & $\begin{array}{l}\operatorname{Cr}(V I), \\
\text { Hach }\end{array}$ & $\mathrm{Cr}_{\mathrm{T}}, \mathrm{ICP}$ \\
\hline Seep 153-1 & $\begin{array}{l}3 / 17 / 93 \\
08: 30\end{array}$ & - & $\begin{array}{l}0.03 \text { uf } \\
0.05 \text { uf }\end{array}$ & $\begin{array}{l}0.045 \text { uf B08BDI } \\
0.050 f \quad \text { B08BD2 }\end{array}$ \\
\hline Seep 110-1 & $\begin{array}{l}3 / 26 / 93 \\
10: 00 \\
\end{array}$ & -- & 0.14 uf & $\begin{array}{ll}0.276 \text { uf } & \text { B08BD7 } \\
0.247 f & \text { B08BD8 } \\
\end{array}$ \\
\hline Seep 110-1 & $\begin{array}{l}3 / 31 / 94 \\
09: 15 \\
\end{array}$ & $<0.05$ В0ВМЈ8 & 0.03 uf & $\begin{array}{ll}0.026 \text { uf } & \text { BOBMJ6 } \\
0.025 f & \text { BOBMJ7 } \\
\end{array}$ \\
\hline Seep 110-1 & $\begin{array}{l}4 / 1 / 94 \\
08: 00\end{array}$ & $\angle 0.05$ BOBMKI & 0.01 uf & $\begin{array}{l}0.025 \text { uf BOBMJg } \\
0.016 \mathrm{f} \text { BOBMKO }\end{array}$ \\
\hline Wel1 Pt \#5 & $\begin{array}{l}4 / 1 / 94^{\circ} \\
09: 00^{\circ}\end{array}$ & $<0.05$ BOBMK 4 & $0.01 \mathrm{f}$ & $\begin{array}{r}12.7 \text { uf BOBMK2 } \\
-0.022 \mathrm{f} \text { BOBMK3 } \\
\end{array}$ \\
\hline $\begin{array}{l}\text { Seep 110-1 } \\
\text { (dup) }\end{array}$ & $\begin{array}{l}4 / 1 / 94 \\
9: 45 \\
\end{array}$ & <0.05 BOBMK & $\begin{array}{l}0.02 \mathrm{f} \\
0.02 \mathrm{uf}\end{array}$ & $\begin{array}{l}0.020 \text { uf BOBMK5 } \\
0.021 \mathrm{f} \text { BOBMK6 } \\
\end{array}$ \\
\hline $\begin{array}{l}\text { Equipment } \\
\text { Blank }\end{array}$ & $\begin{array}{l}4 / 1 / 94 \\
11: 30 \\
\end{array}$ & $<0.05$ BOBMLO & $0.00 f$ & $\begin{array}{ll}\leq 0.003 \text { uf } & \text { BOBMK8 } \\
\leq 0.004 \mathrm{f} & \text { BOBMKg }\end{array}$ \\
\hline
\end{tabular}

Hexavalent and Total Chromium Analytical Data (mg/L) and Conductivity $(\mu \mathrm{s} / \mathrm{cm})$ of Drive Point Water Samples Collected at 100-D.

\begin{tabular}{|c|c|c|c|c|}
\hline Location & $\begin{array}{l}\text { Date } \\
\text { Time }\end{array}$ & $\begin{array}{l}\operatorname{Cr}(V I) \\
\text { WHC } 7196\end{array}$ & $\begin{array}{l}\mathrm{Cr}_{\mathrm{T}}, \\
\mathrm{Hach}\end{array}$ & Cond, $\mu \mathrm{s} / \mathrm{cm}$ \\
\hline Well Pt \#1 & $\begin{array}{l}3 / 31 / 94 \\
10: 15 \\
\end{array}$ & $\begin{array}{l}0.08 \text { uf } \\
0.09 \text { uf }\end{array}$ & $\begin{array}{l}0.12 u f \\
0.13 u f \\
\end{array}$ & $\begin{array}{l}183 \\
188 \\
\end{array}$ \\
\hline Wel1 Pt \#2 & $\begin{array}{l}3 / 31 / 94 \\
10: 30 \\
\end{array}$ & $\begin{array}{l}0.06 \text { uf } \\
0.06 \text { uf }\end{array}$ & $\begin{array}{l}0.14 \text { uf } \\
0.14 \text { uf }\end{array}$ & $\begin{array}{l}187 \\
188 \\
\end{array}$ \\
\hline We11 Pt \#3 & $\begin{array}{l}3 / 31 / 94 \\
10: 35\end{array}$ & $\begin{array}{l}0.04 \text { uf } \\
0.04 \text { uf }\end{array}$ & $\begin{array}{l}0.12 \text { uf } \\
0.14 \text { uf } \\
\end{array}$ & $\begin{array}{l}177 \\
176 \\
\end{array}$ \\
\hline Well Pt $\ddot{\pi} 4$ & $\begin{array}{l}3 / 31 / 94 \\
10: 40 \\
\end{array}$ & $\begin{array}{l}0.00 \text { uf } \\
0.00 \text { uf }\end{array}$ & $\begin{array}{l}0.10 \text { uf } \\
0.09 \text { uf }\end{array}$ & $\begin{array}{l}145 \\
144 \\
\end{array}$ \\
\hline We11 Pt \#5 & $\begin{array}{l}3 / 31 / 94 \\
11: 00 \\
\end{array}$ & $\begin{array}{l}0.02 \text { uf } \\
0.03 \text { uf }\end{array}$ & $\begin{array}{l}0.10 \text { uf } \\
0.10 \text { uf }\end{array}$ & $\begin{array}{l}155 \\
157 \\
\end{array}$ \\
\hline Well Pt \#6 & $\begin{array}{l}3 / 31 / 94 \\
11: 10\end{array}$ & $\begin{array}{l}0.10 \text { uf } \\
0.10 \text { uf }\end{array}$ & $\begin{array}{l}0.18 \text { uf } \\
0.18 \text { uf }\end{array}$ & $\begin{array}{l}193 \\
193\end{array}$ \\
\hline
\end{tabular}




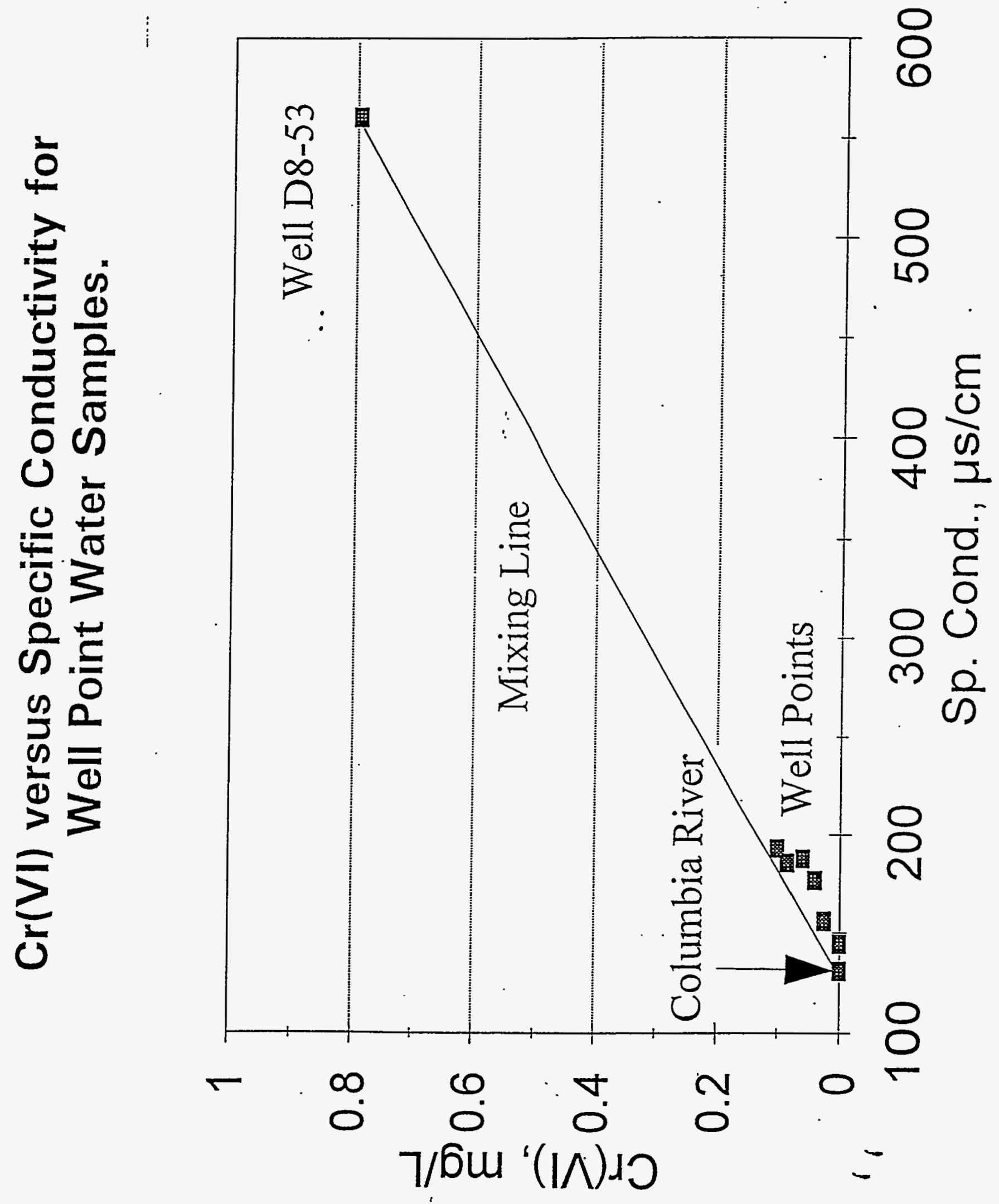




\section{RESULTS OF CHROMIUM SPECIATION STUDIES}

- Chromium Speciation of Groundwater Samples

- Elevated chromium in filtered samples represents dissolved hexavalent chromium

- Unfiltered samples often contain reduced chromium associated with particulate matter

- Chromium Speciation of Seep and Drive Point Samples

- Dissolved chromium is predominantly in the hexavalent oxidation state

- Data suggests Cr(VI) is conserved during groundwater/ river mixing

- Chromium Speciation of Columbia River Samples

- Total dissolved chromium $\sim 0.25 \mathrm{ppb}$ at 100D

,- Total dissolved chromium $0.55 \mathrm{ppb}$ near $100 \mathrm{H}$, - 80\% Cr(VI) 


\section{CHARACTERIZATION OF RIVERBANK SEDIMENTS}

- Approach and Objectives

- Four sediment samples collected near seep 110-1 of 100D Area

- Determine concentration and speciation of chromium in sediments

- Assess interaction processes between riverbank sediments and groundwater

- Characterization and Testing Methods

- Mineralogical/textural analyses by XRD

- Semi-quantitative PIXE chemical analysis

- Extraction and dissolution tests

- Batch sediment/solution interaction tests 


\section{RESULTS OF SEDIMENT STUDIES}

- Sediment Characterization Results

- Texturally sands with minor clay/silt fraction

- Composed primarily of quartz and feldspar plus kaolin/serpentine, mica/illite, and smectite

- Moderately enriched in chromium (85 to $145 \mathrm{ppm}$ )

\& Implications

- Minor uptake of chromium by sediment occurs as groundwater passes through riverbank

- Chromium precipitates as a result of reduction by labile iron and organic matter in sediments

- Most chromium probably passes through riverbank mixing zone relatively unaltered, however 


\section{GROUNDWATER/RIVER MIXING STUDY}

- Objective

- Evaluate potential changes in speciation or concentration of dissolved chromium as groundwater discharges into the Columbia River

ì - Approach

- River water composite prepared from samples collected at 10 locations ( $\sim 40$ liters)

- Composite mixed with chromate-contaminated groundwater from well D5-15 at various ratios

- Controls and mixtures analyzed for $\operatorname{Cr}(\mathrm{VI})$ and other constituents at the beginning of each test and at 4 hours, 1 day, 3 days, 7 days, and 21 days 


\section{Sampling Locations for Columbia River Composite.}

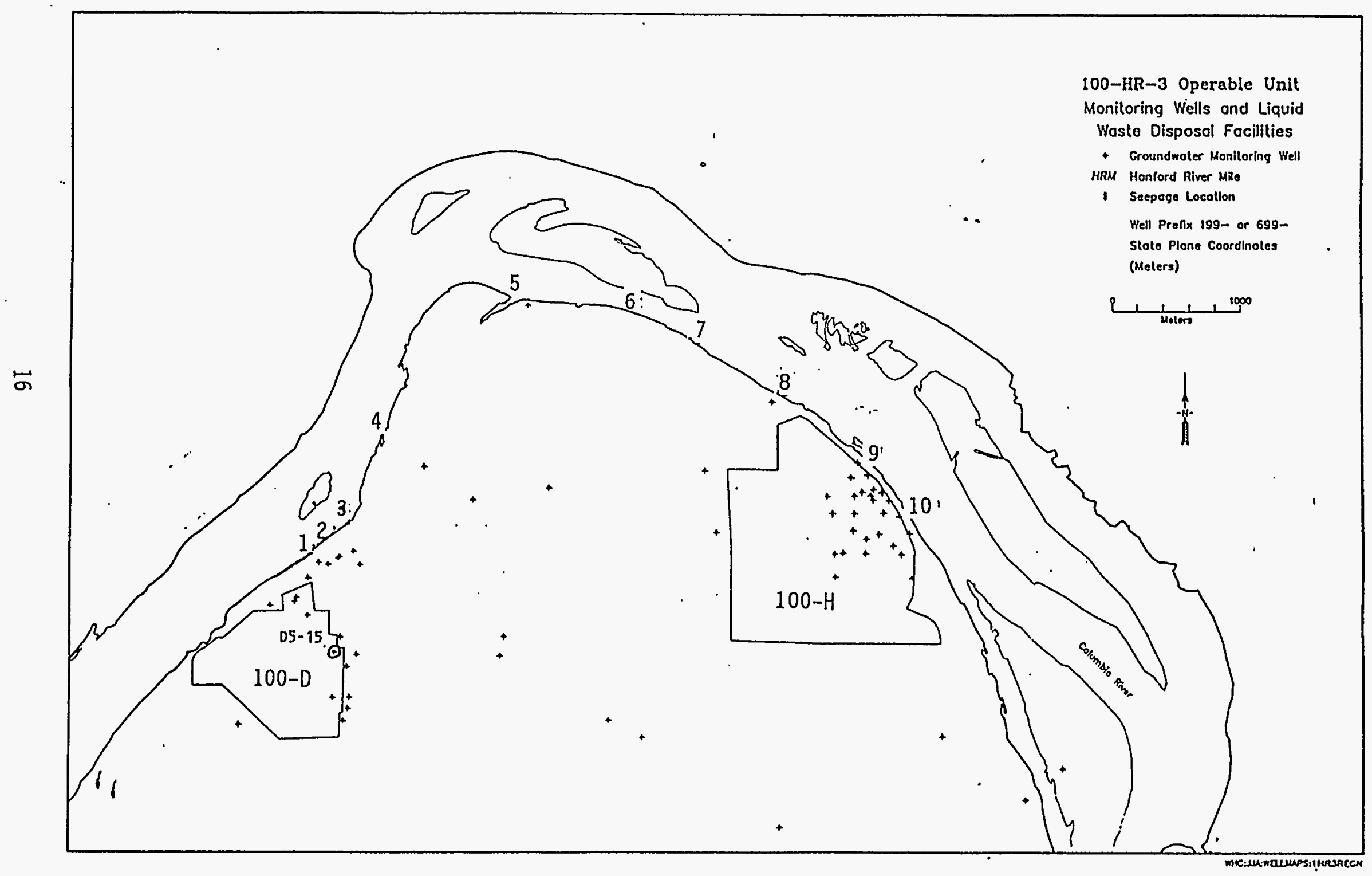




\section{DESCRIPTION OF TESTS PERFORMED}

Test 1 - River water control (no chromium)

Test 2 - River/groundwater ratio $=1$

Test 3 - River/groundwater ratio $=10$

Test 4 - River/groundwater ratio $=100$

Test 5 - River/groundwater ratio $=\mathbf{5 0 0}$

Test 6 - River/groundwater ratio $=10$, mixture exposed to UV light to evaluate photooxidative effects

${ }^{5}$ Test $7-2000 \mathrm{ppb} \mathrm{Cr}(\mathrm{VI})$ standard solution added to river water at a river/solution ratio $=10$ to evaluate groundwater matrix effects

Test 8 - D5-15 groundwater control, 1600 ppb Cr(VI)

Test 9 - Filtered groundwater and river water combined at a river/groundwater ratio $=10$ to evaluate effect of suspended particulate matter on $\mathrm{Cr}(\mathrm{VI})$ concentration 


\section{Cr(VI) Concentration versus Time for Mixing Tests.}

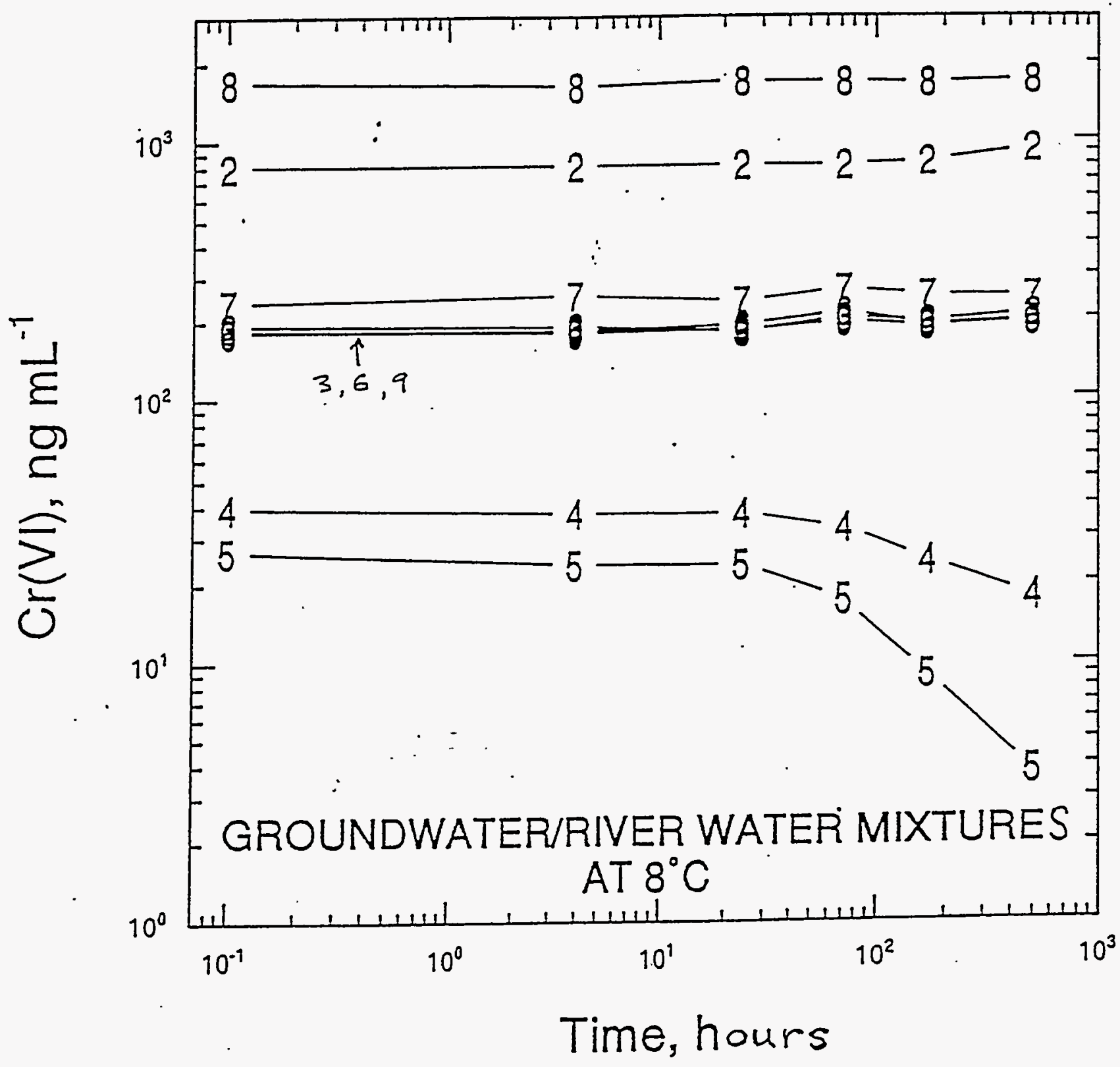




\section{RESULTS OF MIXING TESTS}

- Principal Observations

- Little change observed in chromium concentration or speciation during laboratory mixing tests conducted at lower river/groundwater ratios

- Possible decrease observed in chromium concentration $\overline{\text { Fे }}$ during tests conducted at higher ratios

- Implications

- Chromium will remain largely in the hexavalent state after entering the Columbia River

- Chromium concentration decreases in river primarily in proportion to dilution, although some reduction and precipitation probably occurs at high dilutions 


\section{CONCLUSIONS}

- Dissolved chromium present in groundwater and seeps is primarily in the hexavalent oxidation state.

- A minor portion of chromium is reduced and precipitated on riverbank sediments as groundwater passes through

$\approx \quad$ the groundwater/river interface, but this is unlikely to be an important sink for chromium.

- Chromium remains in the hexavalent oxidation state after groundwater mixes with the Columbia River and varies in concentration primarily in proportion to the degree of dilution. 
WHC-SA-2925-FP

DISTRIBUTION

Number of copies

ONSITE

3

Hestinghouse Hanford Company

E. C. Thornton

H6-06

Information Release

Administration

Central Files

H6-08

A3-88

Distr-1 